\title{
Axillary artery cannulation for cardiopulmonary bypass reduces cerebral microemboli
}

Nasim Hedayati, MD

J. Timothy Sherwood, MD

Steve J. Schomisch, BS

Joseph L. Carino, BS

Alan H. Markowitz, MD

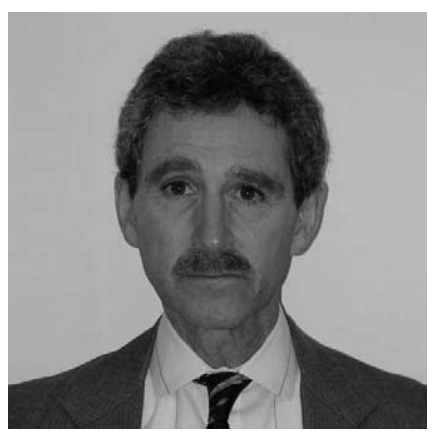

Dr Markowitz
From the Division of Cardiothoracic Surgery, Case Western Reserve University/ University Hospitals of Cleveland, Cleveland, Ohio.

Dr Hedayati is an Allen Fellow supported by the Jay L. Ankeney Endowed Professorship in Cardiothoracic Surgery, Case Western Reserve University School of Medicine. This project was supported by the Jay L. Ankeney Endowment, with partial funding by Medtronic Inc (Minneapolis, Minn).

Received for publication Aug 7, 2003; revisions requested Nov 14, 2003; revisions received Jan 8, 2004; accepted for publication Jan 28, 2004.

Address for reprints: Alan H. Markowitz, MD, University Hospitals of Cleveland, Cardiothoracic Surgery, 11100 Euclid Ave, Cleveland, OH 44106-5011 (E-mail: alan. markowitz@uhhs.com).

J Thorac Cardiovasc Surg 2004;128:386-90 $0022-5223 / \$ 30.00$

Copyright () 2004 by The American Association for Thoracic Surgery

doi:10.1016/j.jtcvs.2004.01.013
Background: Aortic cannulation for cardiopulmonary bypass (CPB) is linked to cerebral microemboli emanating from the ascending aorta. Aortic calcification or disease requiring replacement precludes aortic cannulation. Clinical experience with axillary artery cannulation led to the hypothesis that axillary cannulation may be cerebroprotective.

Methods: Five mongrel dogs underwent a median sternotomy and isolation of the right axillary artery. The canine bicarotid brachiocephalic trunk was reconfigured by grafting the origin of the left carotid to the proximal left subclavian artery. Microspheres were injected into the ascending aorta during 4 conditions: before and after reconfiguration, CPB with aortic cannulation, and CPB with axillary cannulation. Brain, kidneys, and skeletal muscle were analyzed for microsphere distribution.

Results: Each animal served as its own control for comparison of aortic and axillary cannulation. No significant differences were documented in microsphere deposition for prereconfiguration and postreconfiguration. In the right middle cerebral artery distribution, $2300 \pm 710$ microspheres per gram were deposited during aortic cannulation, compared with $540 \pm 110$ during axillary cannulation $(P<.05)$. In the left middle cerebral artery region, $2030 \pm 330$ microspheres per gram with aortic cannulation were reduced to $1320 \pm 240$ with axillary cannulation $(P<.05)$. Axillary cannulation resulted in $73 \%$ fewer microspheres in the right brain and $40 \%$ fewer microspheres in the left compared with aortic cannulation $(P<.05)$.

Conclusions: Axillary artery cannulation for $\mathrm{CPB}$ is cerebroprotective. Altered blood-flow patterns during axillary cannulation may produce retrograde brachiocephalic artery blood flow and competing intracerebral right-to-left collateral blood flow, deflecting emboli from the ascending aorta and arch toward the descending aorta. Expanded use of axillary artery cannulation during cardiac operations could decrease the incidence of stroke.

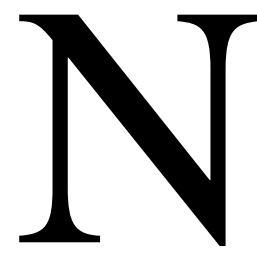

eurologic complications remain a primary cause of morbidity and mortality after cardiac operations. ${ }^{1-3}$ Perioperative stroke, defined as neurologic deficit that occurs within 30 days of operation and persists longer than 1 week, occurs in $1.5 \%$ to $6.1 \%$ of patients. ${ }^{1,4,5}$ The mortality rate of patients who experience a stroke after cardiac operations has been reported at $20 \%$ to $38 \%$. $^{1,2,5,6}$ Activation of systemic inflammatory response mediators, the presence of gaseous and/or particulate emboli in the cardiopulmonary bypass (CPB) circuit, and cerebral hypoperfusion have been implicated in neurologic dysfunction after cardiac operations. ${ }^{2,3}$ The most important cause of neurologic dysfunction after CPB, however, is believed to be cerebral emboli, ${ }^{4,7}$ specifically aortic atheroemboli released during aortic cannulation or decannulation for CPB. ${ }^{8}$ 
The ascending aorta has been the primary route of return for CPB since the early 1970s. Atherosclerosis or aneurysm of the ascending aorta, however, can preclude its cannulation, necessitating the use of other sites. One alternativecannulation of the femoral artery-elicits retrograde perfusion through the descending aorta, which is likely to be atherosclerotic, limiting the use of this technique. ${ }^{9}$ The axillary artery, however, is rarely diseased and benefits from rich collateral blood flow about the shoulder. ${ }^{9}$ When cannulated, it generates antegrade blood flow through the descending aorta. Axillary artery cannulation has been beneficial for the repair of type A aortic dissection, ${ }^{9}$ ascending aorta aneurysm, ${ }^{10}$ and coronary artery disease in cases of severe aortic calcification ${ }^{11}$ and demonstrates few complications secondary to the technique. ${ }^{12}$ Additionally, our experience found that axillary artery cannulation provided a reproducible, neurologically safe approach in a group of patients at high risk for intraoperative neurologic morbidity. ${ }^{13}$ Despite minimal neurologic sequelae reported, ${ }^{9,11,13}$ axillary artery cannulation has yet to gain widespread use in cardiac surgery. The purpose of this study was to contrast the cerebral distribution of microemboli resulting from traditional aortic cannulation with that of axillary artery cannulation during CPB. We proposed that the blood-flow dynamics during $\mathrm{CPB}$ after axillary artery cannulation would result in significantly fewer microemboli in the cerebral circulation than after aortic cannulation.

\section{Methods}

This study was approved by the Case Western Reserve University Institutional Animal Care and Use Committee and conformed to the Guide for the Care and Use of Laboratory Animals prepared by the Institute of Laboratory Animal Resources, National Research Council, and published by the National Academy Press (revised 1996). Six male mongrel dogs $(25-35 \mathrm{~kg})$ were used for the study. One animal died because of surgical complications before CPB. Anesthesia was induced with thiopental sodium $(25 \mathrm{mg} / \mathrm{kg}$ intravenously [IV]) and maintained with fentanyl $\left(3 \mu \mathrm{g} \cdot \mathrm{kg}^{-1} \cdot \mathrm{min}^{-1}\right)$ and midazolam $\left(9.6 \mu \mathrm{g} \cdot \mathrm{kg}^{-1} \cdot \mathrm{min}^{-1}\right)$. Prophylactic cefazolin $(1 \mathrm{~g})$ was administered, and lactated Ringer solution was delivered at $50 \mathrm{~mL} / \mathrm{h}$ (IV). Heart rate, oxygen saturation, mean arterial blood pressure (MAP), central venous pressure, and temperature were continuously monitored. The animals were intubated $(8.0 \mathrm{~F})$ and mechanically ventilated with $100 \%$ oxygen at 12 breaths per minute and a tidal volume of $15 \mathrm{~mL} / \mathrm{kg}$. Paralysis was achieved with pancuronium bromide $(1 \mathrm{mg})$ before chest incision.

The femoral artery and vein were isolated through a left groin incision. A $6 \mathrm{~F}$ venous catheter was placed in the femoral vein to monitor central venous pressure and administer IV fluids and medications. The femoral artery was cannulated with a $6 \mathrm{~F}$ catheter to monitor MAP and sample peripheral blood to determine regional blood flow, blood gas values, and hematocrit. Distal to the arterial catheter placement, a 4- to 5-cm portion of the artery was harvested for later graft use. Through a right-sided midaxillary incision, the axillary artery was isolated. The left and right carotid arteries were mobilized through a midline neck incision and fitted with an ultrasonic flow probe (Transonic Systems Inc, Ithaca, NY) for measurement of carotid blood flow.

A median sternotomy was performed, and the pericardium was opened. The aortic arch was dissected, and the brachiocephalic, left subclavian, and carotid arteries were mobilized. A needle vent was introduced into the ascending aorta for injection of fluorescent microspheres $\left(15-\mu \mathrm{m}\right.$ diameter; $2.5 \times 10^{6}$ particles per milliliter; Interactive Medical Technologies Ltd, Irvine, Calif). A different color was used for each injection to distinguish the conditions. The microspheres were mixed immediately before injection to ensure a homogenous suspension. Heart rate, temperature, $\mathrm{MAP}, \mathrm{PCO}_{2}$, and hematocrit were manipulated to ensure similar values between comparative conditions. Immediately after each injection, peripheral blood was sampled from the femoral artery for calculation of sphere concentration.

The canine aortic arch has 2 main branches: the brachiocephalic artery, from which both carotids originate, and the left subclavian artery. The left carotid artery was detached from the brachiocephalic artery and anastomosed to the proximal left subclavian artery by using an autologous femoral artery (Figure 1). Our aim was to reproduce the separate carotid origins of primate anatomy by reconfiguring the origin of the left carotid distal to the brachiocephalic artery. Microspheres were injected before and after reconfiguration to ensure that the anatomic alteration alone did not alter the steady-state pattern of cerebral blood flow.

Before cannulation for CPB, heparin (100 U/kg IV) was readministered. The bypass circuit (Pemco Inc, Cleveland, Ohio) was primed with 800 to $1000 \mathrm{~mL}$ of Plasmalyte solution (Baxter Healthcare Corp, Deerfield, Ill) along with heparin, $\mathrm{NaHCO}_{3}$, and albumin. The ascending aorta and the right axillary artery were cannulated with a $10 \mathrm{~F}$ pediatric arterial cannula (Medtronic, Grand Rapids, Mich), and venous return was established with a right atrial 32F catheter (Sarns; 3M Health Care Group, Ann Arbor, Mich). The aortic root was crossclamped, and fibrillatory arrest was established. During CPB, the MAP was maintained at $60 \pm 8$ $\mathrm{mm} \mathrm{Hg}$ by administering phenylephrine hydrochloride and norepinephrine bitartrate as required and by adjusting the blood-flow rate (1.9 to $3.4 \mathrm{~L} \cdot \mathrm{min}^{-1} \cdot \mathrm{m}^{-2}$ ). Microsphere injections were repeated during bypass via aortic and axillary cannulation in a randomized order in each animal. Blood samples were collected immediately after each injection as previously described.

At the conclusion of the experiment, the animals were killed with saturated potassium chloride. The brain, kidneys, and a segment of skeletal muscle were removed and stored in aqueous buffered zinc formalin for 7 days. Tissue samples from the distribution of the middle cerebral arteries (MCAs; 2.18-6.50 g) which included the temporal lobes and the inferior portion of the frontal lobes, the occipital lobes (1.73-6.10 g), the kidneys (3.36$12.80 \mathrm{~g})$, and the quadriceps muscle (2.90-10.97 g) —were obtained and processed (Interactive Medical Technologies Ltd) to determine microsphere distribution (number per gram of tissue). Tissue and blood samples containing microspheres were first digested by potassium hydroxide to recover the microspheres and were subsequently separated by negative pressure filtration or sedimentation. Organic solvent was then added to extract the fluorescent dye from the microspheres, and the fluorescent signal from each sample was measured by a 96-well plate reader or an 

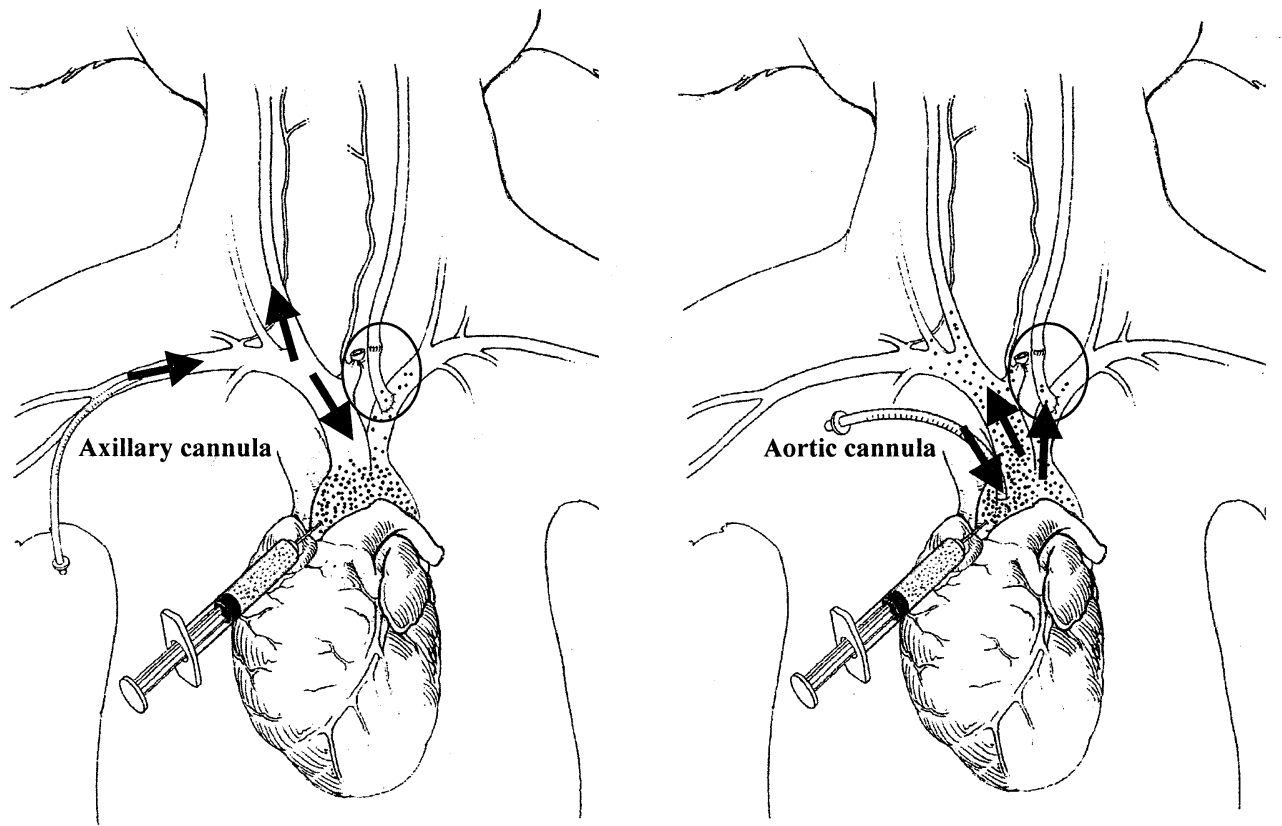

Figure 1. Reconfiguration of the canine bicarotid trunk and cannulation for cardiopulmonary bypass. Carotid origins were separated by grafting the left carotid artery to the left subclavian artery with the autologous femoral artery (circled). Microspheres were injected into the ascending aorta during axillary cannulation (left) and aortic cannulation (right) with predicted flow patterns (arrows).

automated flow cell. Total fluorescence was then converted to represent the quantity of spheres.

All values are expressed as mean $\pm \mathrm{SD}$. Each animal served as its own control for comparison of prereconfigured and postreconfigured conditions and for aortic cannulation versus axillary cannulation. Results were compared between before and after reconfiguration and between aortic and axillary cannulation by using paired Student $t$ tests.

\section{Results}

Five animals completed the experimental protocol. No significant differences were present in MAP $(P=.498)$, temperature $(P=.054), \mathrm{PCO}_{2}(P=.398)$, or hematocrit $(P=$ .484) before and after reconfiguration. Furthermore, the distribution of microspheres in the cerebral and peripheral tissue was not statistically different between these 2 conditions (Table 1).

Physiologic parameters did not vary between axillary and aortic cannulation $(P>.800$ for all). Blood flow in the right carotid artery was $51.4 \pm 36.0 \mathrm{~mL} / \mathrm{min}$ during axillary cannulation and $50.6 \pm 41.9 \mathrm{~mL} / \mathrm{min}$ during aortic cannulation $(P=.803)$. Similarly, blood flow in the left carotid artery was not altered by the cannulation technique (axillary, $39.8 \pm 23.7 \mathrm{~mL} / \mathrm{min}$; aortic, $45.7 \pm 38.1 \mathrm{~mL} / \mathrm{min} ; P=$ .455). Significant differences in microsphere distribution were noted, however, between the 2 CPB cannulation techniques (Figure 2 and Table 2). In the left MCA distribution, $2030 \pm 330$ microspheres per gram were deposited with aortic cannulation and $1320 \pm 240$ microspheres per gram with axillary cannulation $(P<.05)$. The left occipital lobe demonstrated $2020 \pm 540$ microspheres per gram during aortic perfusion and $1080 \pm 360$ microspheres per gram during axillary perfusion $(P<.05)$. During aortic cannulation, $2300 \pm 710$ microspheres per gram were deposited in the right $\mathrm{MCA}$ distribution, compared with $540 \pm 110$ microspheres per gram with axillary cannulation $(P<.05)$. Finally, in the right occipital lobe, $2290 \pm 610$ microspheres per gram were deposited with aortic perfusion versus $650 \pm 230$ microspheres per gram with axillary perfusion $(P<.05)$.

The distribution of microspheres in the peripheral tissues during aortic and axillary cannulation is presented in Table 2. Although no significant differences were found in the blood or muscle between these 2 conditions, significantly more microspheres were deposited in the kidneys with axillary cannulation than with aortic cannulation.

\section{Discussion}

The axillary artery has been successfully cannulated when severe calcification or aneurysm of the aorta have precluded cannulation of the ascending aorta and arch. ${ }^{8,10,11}$ Although there are rare reports of axillary artery thrombosis, brachial plexus injury, or dissection of the aorta secondary to axillary artery cannulation, ${ }^{8,10,12}$ the axillary artery is rarely diseased and can be safely cannulated directly or via a graft 


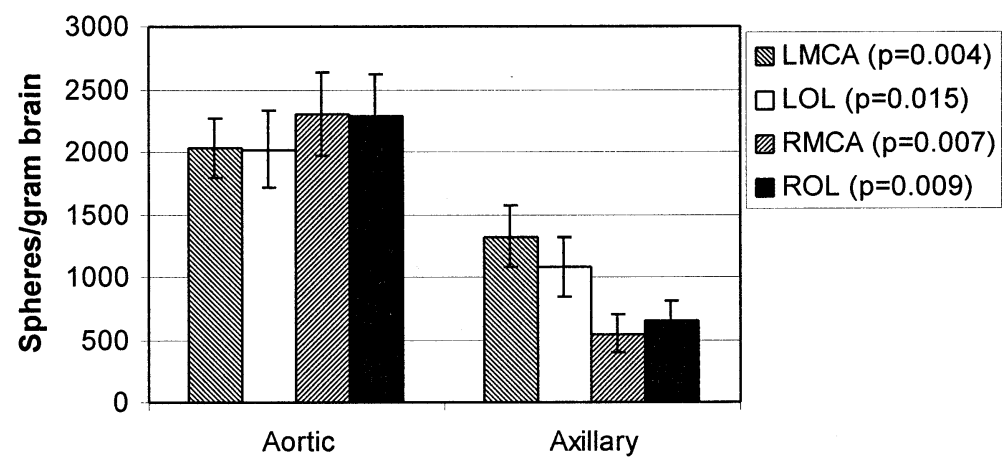

Figure 2. Cerebral microsphere distribution, expressed as mean \pm SD for aortic and axillary cannulation $(n=5)$, indicating fewer spheres in the cerebral circulation during axillary cannulation. $L M C A$, Left middle cerebral artery; $R M C A$, right middle cerebral artery; $L O L$, left occipital lobe; $R O L$, right occipital lobe.

TABLE 1. Microsphere distribution before and after anatomic reconfiguration

\begin{tabular}{lccc}
\hline Tissue & Before reconfiguration & After reconfiguration & $P$ value \\
\hline Left MCA & $590 \pm 240$ & $750 \pm 250$ & .366 \\
Left OL & $670 \pm 310$ & $780 \pm 240$ & .527 \\
Right MCA & $660 \pm 330$ & $670 \pm 150$ & .843 \\
Right OL & $710 \pm 330$ & $730 \pm 160$ & .700 \\
Muscle & $490 \pm 850$ & $450 \pm 780$ & .340 \\
Left kidney & $9560 \pm 3800$ & $11,080 \pm 4390$ & .314 \\
Right kidney & $10,140 \pm 4680$ & $10,730 \pm 3870$ & .715 \\
Blood & $3130 \pm 1270$ & $2890 \pm 430$ & .699 \\
\hline
\end{tabular}

Data are mean \pm SD. $M C A$, Middle cerebral artery; $O L$, occipital lobe.

with an end-to-side anastomosis. Axillary artery cannulation not only avoids aortic manipulation during cannulation but also avoids the retrograde flow of femoral artery cannulation and the resulting "sandblasting" effect of flow in an atherosclerotic aorta. ${ }^{11}$ Possible contraindications to cannulation of the axillary artery would be extension of aortic disease into the artery or axillary/subclavian artery stenosis.

To our knowledge, no previous study has examined the effect of cannulation sites for CPB on the incidence of cerebral microemboli. In this study, we reconfigured the bicarotid trunk of the dog to mimic primate anatomy. With the left carotid artery relocated distal to the retrograde blood flow in the brachiocephalic artery, we ensured that the left carotid artery distribution would not be preferentially protected from the microspheres during axillary perfusion, and we confirmed that this alteration did not affect microsphere distribution.

In the regions supplied by the MCA, where most strokes after cardiac operations have been documented, ${ }^{4}$ and in the region of the occipital lobes, we demonstrated significantly fewer microspheres during axillary perfusion than during aortic perfusion. Overall, we found that axillary artery perfusion resulted in $73 \%$ fewer microspheres deposited in the right brain and $40 \%$ fewer in the left brain when compared with ascending aorta perfusion. Blood flow in the left and
TABLE 2. Microsphere distribution for aortic and axillary cannulation

\begin{tabular}{lccc}
\hline Tissue & Aortic cannulation & Axillary cannulation & $\boldsymbol{P}$ value \\
\hline Left MCA & $2030 \pm 330$ & $1320 \pm 240$ & .004 \\
Left OL & $2020 \pm 540$ & $1080 \pm 360$ & .015 \\
Right MCA & $2300 \pm 710$ & $540 \pm 110$ & .007 \\
Right OL & $2290 \pm 610$ & $650 \pm 230$ & .009 \\
Muscle & $350 \pm 720$ & $580 \pm 1160$ & .320 \\
Left kidney & $3950 \pm 3970$ & $7910 \pm 4220$ & .034 \\
Right kidney & $4270 \pm 4210$ & $8160 \pm 4150$ & .004 \\
Blood & $2320 \pm 1780$ & $4980 \pm 2830$ & .186 \\
\hline
\end{tabular}

Data are mean $\pm S D$. MCA, Middle cerebral artery; $O L$, occipital lobe.

right carotid arteries was unchanged between axillary and aortic cannulation, indicating that the reduction in microspheres during axillary cannulation was not due to diminished blood flow to the brain. Additional support of the hypothesis that the microspheres are deflected from entering the cerebral circulation during axillary perfusion is that more microspheres were deposited in the peripheral tissues, especially the kidneys. These results support the consideration that axillary artery cannulation can decrease the risk of cerebral microembolization during CPB. Whether the cerebroprotective effect witnessed with axillary cannulation is 
dependent on altered blood-flow dynamics in the brachiocephalic artery and aortic arch or on cross-circulation via the circle of Willis is not discernible in this study and will be the subject of future investigations.

In conclusion, we provide evidence that during $\mathrm{CPB}$ with axillary artery cannulation, significantly fewer microspheres (originating in the ascending aorta) are deposited in the brain than with aortic cannulation. Wider application of axillary artery cannulation for cardiac surgery may reduce the risk of perioperative stroke.

We thank Jose Suarez, MD, Osama Zaidat, MD, and Andy Wright for their technical assistance with the tissue processing. We also thank Jeff Foster and Anson Casille for operating the perfusion pump.

\section{References}

1. Roach GW, Kanchuger M, Mora Mangano C, Newman M, Nussmeier $\mathrm{N}$, Wolman R, et al. Adverse cerebral outcomes after coronary bypass surgery. N Engl J Med. 1996;335:1857-63.

2. Seines OA, Goldsborough MA, Borowicz LM, McKhann GM. Neurobehavioural sequelae of cardiopulmonary bypass. Lancet. 1999;353: 1601-5.

3. Restreop L, Wityk RJ. Stroke after coronary bypass surgery: Stroke Clinic Update. Natl Stroke Assoc. 2002;12(2):1-5.

4. Borger MA, Ivanov J, Weisel RD, Rao V, Peniston CM. Stroke during coronary bypass surgery: principal role of cerebral macroemboli. Eur J Cardiothorac Surg. 2001;19:627-32.

5. Calafiore AM, Di Mauro M, Teodori G, Di Giammarco G, Cirmeni S, Contini $\mathrm{M}$, et al. Impact of aortic manipulation on incidence of cerebrovascular accidents after surgical myocardial revascularization. Ann Thorac Surg. 2002;73:1387-93.

6. McKhann GM, Goldsborough MA, Borowicz LM, Mellits ED, Brookmeyer R, Quaskey SA, et al. Predictors of stroke risk in coronary bypass patients. Ann Thorac Surg. 1997;63:516-21.

7. Raymond PD, Marsh NA. Alterations to haemostasis following cardiopulmonary bypass and the relationship of these changes to neurocognitive morbidity. Blood Coagul Fibrinolysis. 2001;12:601-18.

8. Fearn SJ, Pole R, Burgess M, Ray SG, Hooper TL, McCollum CN. Cerebral embolism during modern cardiopulmonary bypass. Eur J Cardiothorac Surg. 2001;20:1163-7.

9. Neri E, Massetti M, Capannini G, Carone R, Tucci E, Diciolla F, et al. Axillary artery cannulation in type A aortic dissection operations. J Thorac Cardiovasc Surg. 1999;118:324-9.

10. Baribeau YR, Westbrook BM, Charlesworth DC, Maloney CT. Arterial inflow via an axillary artery graft for the severely atheromatous aorta. Ann Thorac Surg. 1998;66:33-7.

11. Sabik JF, Lytle BW, McCarthy PM, Cosgrove DM. Axillary artery: an alternative site of arterial cannulation for patients with extensive aortic and peripheral vascular disease. J Thorac Cardiovasc Surg. 1995;109: 885-91.

12. Miyatake T, Matsui Y, Suto Y, Imamura M, Shiya N, Murashita T, et al. A case of intraoperative acute aortic dissection caused by cannulation into an axillary artery. J Cardiovasc Surg. 2001;42:809-11.

13. Markowitz A. Utility of the full root bioprosthesis in surgery for complex aortic valve-ascending aortic disease. Semin Thorac Cardiovasc Surg. 2001;13(suppl 1):12-15.

\section{JTCVS On-Line Manuscript Submission and Review}

\section{Please visit http://www.editorialmanager.com/jtcvs/}

Effective September 15, 2001, authors and reviewers may submit manuscripts and reviews electronically via Editorial Manager, our new Web-based system with full electronic submission, review, and status update capabilities.

As we move from paper to electronic submissions, the Editorial Office will make proxy submissions of all manuscripts accompanied by a diskette containing the electronic files of the text, tables, and figures. Editors, authors, and reviewers will receive automatic e-mails when significant events occur.

We strongly encourage all authors and reviewers to use Editorial Manager. Although we will continue to accommodate the submission of paper manuscripts for some months, our goal is to be completely electronic within 9 to 12 months.

All individuals currently in our database for whom we have e-mail addresses will receive via e-mail a system-assigned username and password that can be used to log in to the system without prior registration. All those not receiving the e-mail must register the first time they use the system.

As with any broad systemic change, the conversion to the new system will take some time to complete. We ask your patience as we replace our in-office database with the new system. We also encourage you to take advantage of the speed and efficiency that the new system will provide for us all: editor, author, reviewer, and publisher. 